\title{
Firmicutes Dysbiosis After Chlorhexidine Prophylaxis in Healthy Patients Submitted to Impacted Lower Third Molar Extraction
}

\section{Carlos Augusto das Neves ${ }^{\dagger}$, Carlos Henrique Alves ${ }^{\dagger}$, Natália Conceição Rocha, Karina Ferreira Rizzardi, Karolyne Larissa Russi, Alexandre Augusto Albigiante Palazzi, Thaís Manzano Parisotto and Raquel Girardello* \\ Laboratório de Microbiologia Molecular e Clínica, Universidade São Francisco, Bragança Paulista, Brazil}

OPEN ACCESS

Edited by:

Rodnei Dennis Rossoni,

Sao Paulo State University, Brazil

Reviewed by:

Fabio Rodriguez Sánchez,

KU Leuven, Belgium

Patrícia Paiva Corsetti,

University of José do Rosário Vellano,

Brazil

${ }^{*}$ Correspondence:

Raquel Girardello

raquel.girardello@usf.edu.br

${ }^{\dagger}$ These authors have contributed equally to this work

Specialty section: This article was submitted to

Bacteria and Host,

a section of the journal

Frontiers in Cellular and

Infection Microbiology

Received: 28 April 2021

Accepted: 26 July 2021

Published: 13 August 2021

Citation:

Neves CAd, Alves CH, Rocha NC, Rizzardi KF, Russi KL, Palazzi AAA, Parisotto TM and Girardello R (2021) Firmicutes Dysbiosis After

Chlorhexidine Prophylaxis in Healthy

Patients Submitted to Impacted

Lower Third Molar Extraction.

Front. Cell. Infect. Microbiol. 11:702014.

doi: 10.3389/fcimb.2021.702014
Prophylaxis with antiseptic and antibiotic therapy is common in impacted lower third molar surgeries, despite the lack of consensus among professionals and researchers in the indication for healthy patients. The aim of the present preliminary study was to verify the impact of prophylaxis therapy with antiseptic and antibiotic in healthy patients submitted to impacted lower third molar extraction, according to oral microorganism quantification. Eleven patients submitted to impacted lower third molar extraction, under prophylactic therapy with $0.12 \%$ chlorhexidine and amoxicillin in four experimental phases, were evaluated. Our results showed no significant reduction in total bacteria load, as well as in Bacteroidetes and C. albicans loads in the oral cavity, after prophylactic therapy with antiseptic and antibiotic. On the other hand, there was a significant difference between the Firmicutes levels across the follow-up, and this effect seems to be large $\left(\eta p^{2}=0.94\right)$. Posthoc test demonstrated that the levels of Firmicutes in T1 were higher than T0, T2, and T3, suggesting a microbiota dysbiosis, when $0.12 \%$ chlorhexidine use, which may be responsible for selection of antibiotic-resistant microorganisms. Our results alert for an overuse of antiseptic and antibiotics by dentists and for a better evaluation of the available protocols.

Keywords: personalized odontology, antibiotic prophylaxis, antibiotic resistance, oral microbiota, oral bacteria and fungi

\section{INTRODUCTION}

Third molar extraction is a routine protocol in odontology and diverse techniques for impacted lower third molars are described in the clinical literature, with different degrees of tissue invasion (Kirk et al., 2007; Coulthard et al., 2014). According to Silvestri and Singh (2003), around 65\% of healthy individuals have impacted third molars, poorly positioned or with difficulty in hygiene access, with reduced function and a high rate of associated diseases.

Prophylactic therapy using antibiotics and antiseptics are common; however, some authors agree that, to establish a prophylactic treatment with the use of antibiotics, the risk of infection must be 
significant, regarding the degree of severity, a fact that is not common in extraction of impacted third molars (Lindeboom, 2008; Neves et al., 2020).

There is no consensus among professionals and researchers about the need and the protocol of this therapy in patients without previous infection (Stein et al., 2018; Menon et al., 2019). Microbiological studies are scarce to determine the real role of prophylactic therapy with antimicrobials in healthy patients. Current knowledge around this theme is based mainly in observational studies and systematic reviews (Lodi et al., 2021; Riba-Terés et al., 2021; Yoshida et al., 2021). The aim of this study was to verify the impact of prophylaxis therapy with antiseptic and antibiotic in healthy patients submitted to impacted lower third molar extraction, according to oral microorganism quantification.

\section{MATERIAL AND METHODS}

\section{Patient's Records Evaluation}

Before the selection of patients for the study, a characterization of the patient's profile attending a private dental office in Bragança Paulista-SP/Brazil, from 2016 to 2020, was performed. This way, the rate of infections before the surgery procedures, was estimated by analyzing patient's records.

\section{Oral Microorganism Quantification}

Eleven healthy patients seeking for noninfected impacted lower third molar surgery were selected for oral bacteria quantification by using qPCR. The samples were collected in the same pattern, using a sterile swab (E-swab, Copan), in four different experimental phases: T0 (before surgery), T1 (after $0.12 \%$ chlorhexidine mouthwash, for 3 days), T2 (after amoxicillin $875 \mathrm{mg}$, single dose, $1 \mathrm{~h}$ before surgery, followed by amoxicillin $875 \mathrm{mg}$ every $12 \mathrm{~h}$, for 7 days after surgery), and T3 (28 days after amoxicillin use). The DNA was extracted by using Microbiome DNA kit (ZymoBiomics, CA, USA), according to manufacture recommendation. The DNA concentration from clinical samples was normalized and submitted to qPCR in a 7300 Real-Time System (Applied Biosystems, Foster City, CA, USA) for total bacteria, Firmicutes phylum, Bacteroidetes phylum, and Candida albicans yeast, according to a protocol previously described (Rizzardi et al., 2021). For each sample, $10 \mathrm{ml}$ of the reaction mixture, including $5 \mu \mathrm{l}$ of SYBR Green Power up (Thermo Fisher Scientific, Carlsbad, CA, USA), $2.9 \mu$ of ultrapure water, $1.5 \mu \mathrm{l}$ of DNA sample, and $0.3 \mu \mathrm{l}$ of each primer were used. Amplification and detection were performed according to the following cycles: $2 \mathrm{~min}$ at $50^{\circ} \mathrm{C}, 10 \mathrm{~min}$ at $95^{\circ} \mathrm{C}, 40$ cycles of $15 \mathrm{~s}$ at $95^{\circ} \mathrm{C}$, and $1 \mathrm{~min}$ at $60^{\circ} \mathrm{C}$ (Guo et al., 2008).

Total bacterial DNA extracted from the oral cavity was quantified, based on the 466-bp fragment of conservative sequence of $16 S$ rRNA gene, using specific primers (F: 5'TCCTACGGGAGGCAGCAGT-3' and R: 5'-GGACTACCAG GGTATCTAATCCTGTT-3'), previously described (Nadkarni et al., 2002). Escherichia coli DH5-Alpha was used as control for bacteria quantification standard curve. For Firmicutes phylum quantification, sequences of primers previously described (F: 5'GGAGYATGTGGTTTAATTCGAAGCA-3' and R: 5' AGCTGACGACAACCATGCAC-3') were used (Guo et al., 2008). Clostridium perfringens ATCC 13124 was used as control for Firmicutes standard curve. Forward primer, 5' GGARCATGTGGTTTAATTCGATGAT- $3^{\prime}$ and reverse primer 5'-AGCTGACGACAACCATGCAG-3' were used to quantify Bacteroidetes phylum bacteria, generating a 126-bp amplicon (Guo et al., 2008). Bacteroides fragilis ATCC 25285 was used as positive control for Bacteroidetes phylum. For C. albicans quantification, primers previously reported by Rahn et al. (2016) were used (FP: CCTGTTTGAGCGTCRTTT RP: TCCTCCGCTTATTGATATGC), based on the 229-bp fragment of conservative sequence of $18 S$ rRNA gene.

All primers were evaluated for their specificity in the software BLAST (Basic Local Alignment Search Tool - http:// www.ncbi.nlm.nih. gov/blast/) and have been confirmed to be specific for their purposes. DNA samples of control strains were serially diluted by 10 -fold (five dilution points; $10^{1}-10^{5} \mathrm{ng} / \mathrm{ml}$ ) to generate standard curve for determining the absolute target quantity in samples. More specifically, the software (Sequence Detection Software version 1.3.1, Applied Biosystems, Foster City, CA, USA) measures the amplification of the target in both the standard dilution series and test samples. A standard curve was generated using data from the standard dilution series. According to the standard curve, the software interpolates the absolute quantity of the target in the test samples.

\section{Statistical Approach}

Data were statistically analyzed using the SPSS package for Windows, version 21.0 (SPSS, Inc., Chicago, IL, USA). Data normality was tested using the Shapiro-Wilk test and Levene test, respectively. Variables that violated the premises of analysis of variances were transformed (Samal et al., 1999) to the logarithm of 10 (total bacteria, Bacteroidetes, and Firmicutes data) and for inverse transformation (C. albicans data).

Descriptive statistics considered the median and interquartile range due to the non-Gaussian distribution. Within subjects' effects were obtained using repeated measures analysis of variance (RMANOVA). Assumptions of sphericity were tested using Mauchly's test of sphericity. Greenhouse-Geisser correction and Huynh-Feldt correction were made considering the cut-off point of 0.75 for $\epsilon$ values when the $p$-value of Mauchly's test of sphericity was significant. Bonferroni adjustment of $p$-value corrected the level of significance of $\alpha$, avoiding family-wise error.

\section{RESULTS AND DISCUSSION}

\section{Patients' Profile Submitted to Impacted Lower Third Molar Extraction}

From 2016 to 2020, 172 patients seek for third molar surgery in the private clinic at Bragança Paulista-SP where they were recruited. One hundred and thirty-three patients $(77.3 \%)$ had 
no infection before the surgery; a minor rate of $22.7 \%$ (39 patients) showed infections, which were classified as pericoronitis (37 patients), and abscess of dental origin (two patients). In total, 288 teeth were extracted, being 195 (67.7\%) impacted.

It is important to highlight that the use of antibiotic in patients with previous infection is necessary and is not the purpose of this study. While the major clinical indications for surgery in the present investigation were due to orthodontic treatment [211 teeth $(73.3 \%)]$, the minor were due to carious process or coronary destruction [13 teeth (4.5\%)]. From 39 patients with previous infection, 38 received amoxicillin (875 mg every $12 \mathrm{~h}$ ) together with $0.12 \%$ chlorhexidine mouthwash, for three consecutive days before the dental surgery, followed by amoxicillin $875 \mathrm{mg}$ therapy for 4 days after the dental procedure, to complete a 7-day therapy protocol. One patient needed hospital internment for the abscess treatment before the surgery procedures. This patient received amoxicillin $875 \mathrm{mg}$ and $0.12 \%$ chlorhexidine mouthwash together with metronidazole (500 mg every $12 \mathrm{~h}$ ), for 7 days. According to the literature, this protocol is necessary for anaerobic bacteria infection (Sukegawa et al., 2019; Dallaserra et al., 2020). The 133 patients without previous infection received $0.12 \%$ chlorhexidine mouthwash, for three consecutive days before the surgery; amoxicillin ( $875 \mathrm{mg}$, single dose), $1 \mathrm{~h}$ before surgery; and amoxicillin ( $875 \mathrm{mg}$ every $12 \mathrm{~h}$ ) for 7 days, after the surgery. The patients included in the microbiological analysis were selected from this last group, if they had impacted lower third molar. No patients had postsurgery infection in the evaluated period.

\section{Oral Microorganisms Quantification}

Total bacteria, Bacteroidetes, and C. albicans load did not differ across the follow-up (T0 vs. T1; T0 vs. T2, and T0 vs. T3) (Table $\mathbf{1}$ and Figures 1A, B, D). These findings are not in accordance with the professionals' protocols of using antiseptic or antibiotic prophylaxis to decrease the bacterial load in order to reduce the postsurgery infections (Lacasa et al., 2007; LuacesRey et al., 2010). Despite the fact that no significant changes have been observed in the total bacteria, there was a significant difference between the Firmicutes levels across the follow-up. This effect seems to be large $\left(\eta p^{2}=0.94\right)$. Interestingly, post-hoc test demonstrated that the levels of Firmicutes in T1 (chlorhexidine antiseptic usage) were higher than T0, T2, and T3 (Figure 1C and Table 1). The oral microbiota has microorganisms with diversity of energetic metabolism, including facultative anaerobic and anaerobic species. Coagulase-negative Staphylococcus and viridans group Streptococcus represent the facultative anaerobes, while Fusobacterium spp., Prevotella spp., Porphyromonas spp., Bacteroides fragilis, Clostridium spp., and Treponema spp. represent the aerobic group. Coagulase-negative Staphylococcus, viridans group Streptococcus, and Clostridium spp. compose the Firmicutes phylum, representing a significant part of oral potential pathogens (Robertson and Smith, 2009). Increase in the Firmicutes level may act to favor the selection of the oral pathogens in healthy individuals, since chlorhexidine antiseptic-based mouthwashes are sold without a prescription and, a previous study in our laboratory showed that they are daily used by healthy individuals after tooth brushing (Russi et al., 2020).

The statistical significance observed only for the Firmicutes phylum load after antiseptic usage may be the result of microbial dysbiosis caused by antiseptic therapy. The dysbiotic condition of the microbiota is associated with systemic disorders (Plachokova et al., 2021), and it may induce the selection of antibiotic-resistant strains, which can result in a therapeutic failure in a future infection episode (Khalil et al., 2016; Neves et al., 2020). According to Khalil et al. (2016), a single dose of amoxicillin is sufficient to cause an ecological disturbance in the oral microbiota and induce selection of resistant strains. In addition, studies have been showing a cross-resistance to antibiotics after antiseptic usage (Beier et al., 2021; Garratt et al., 2021; Merchel Piovesan Pereira et al., 2021). Furthermore, even after 28 days after prophylaxis, the oral bacteria quantification did not seem to return to baseline levels (T0 vs. T3), suggesting that the patients may not have been able to reestablish their microbiota after the dysbiosis (Table 1). This is a preliminary study using a convenience sample. The major problem of a convenience

TABLE 1 | Within subjects' effects of antiseptic and antibiotic intervention across the time in total bacteria, Bacteroidetes and Firmicutes Phyla, and C. albicans: a repeated measures analysis of variance.

\begin{tabular}{|c|c|c|c|c|}
\hline Interventions across the time & $\begin{array}{l}\text { Total bacteria } \\
\text { Median (IQR) }\end{array}$ & $\begin{array}{l}\text { Bacteroidetes } \\
\text { Median (IQR) }\end{array}$ & $\begin{array}{c}\text { Firmicutes } \\
\text { Median (IQR) }\end{array}$ & $\begin{array}{c}\text { Candida albicans } \\
\text { Median (IQR) }\end{array}$ \\
\hline TO & $6.27 \times 10^{3}\left(4.04 \times 10^{10}\right)$ & $9.07 \times 10^{6}\left(1.16 \times 10^{28}\right)$ & $1.82 \times 10^{2}\left(8.13 \times 10^{3}\right)$ & $8.15 \times 10^{38}\left(2.70 \times 10^{39}\right)$ \\
\hline$T 1$ & $0.175 \times 10^{2}\left(8.25 \times 10^{7}\right)$ & $2.01 \times 10^{6}\left(2.92 \times 10^{20}\right)$ & $1.06 \times 10^{11}\left(4.38 \times 10^{9}\right)^{\star \star \star}$ & $5.64 \times 10^{16}\left(6.13 \times 10^{12}\right)$ \\
\hline$T 2$ & $1.93 \times 10^{4}\left(2.81 \times 10^{16}\right)$ & $4.94 \times 10^{23}\left(1.93 \times 10^{29}\right)$ & $5.44 \times 10^{3}\left(5.66 \times 10^{3}\right)$ & $6.57 \times 10^{8}\left(2.18 \times 10^{9}\right)$ \\
\hline T3 & $1.30 \times 10^{3}\left(1.14 \times 10^{10}\right)$ & $5.24 \times 10^{16}\left(2.30 \times 10^{26}\right)$ & $4.58 \times 10^{3}\left(8.02 \times 10^{3}\right)$ & $1.67 \times 10^{14}\left(5.55 \times 10^{14}\right)$ \\
\hline \multicolumn{5}{|l|}{ RMANOVA } \\
\hline$p$-Value (power) & $0.387(0.14) \boldsymbol{\beta}$ & $0.396(0.16) \boldsymbol{\beta}$ & $0.000(1.00)$ & $0.101(0.51)$ \\
\hline$\eta p^{2}$ & 0.100 & 0.119 & 0.949 & 0.203 \\
\hline
\end{tabular}

Statistical analyses were performed with a sample of 11 volunteers. $\eta p^{2}$, partial eta squared. Total bacteria, Bacteroidetes, and Firmicutes data were transformed by log 10 to achieve the RMANOVA premises. C. albicans data were reciprocally transformed considering $1 / x$.

$\beta$, significant $p$-value for Mauchly's test of sphericity required the Greenhouse-Geisser correction.

${ }^{* * *}$ Means $p$-value lower than 0.0000 after Bonferroni adjustment for multiple comparisons. 


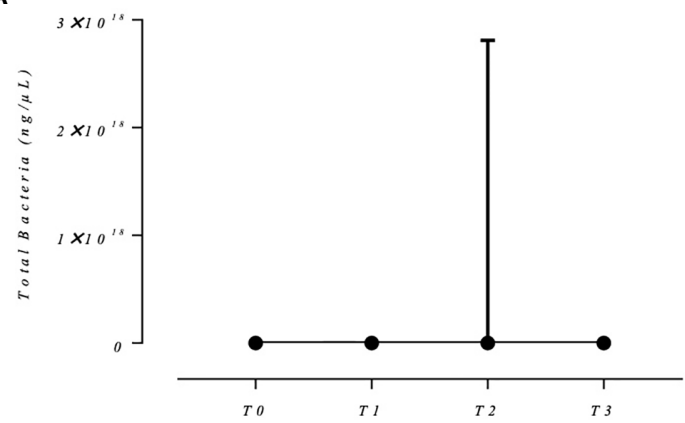

C

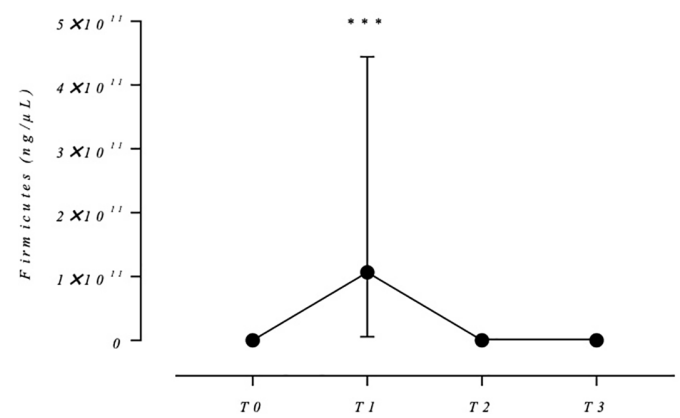

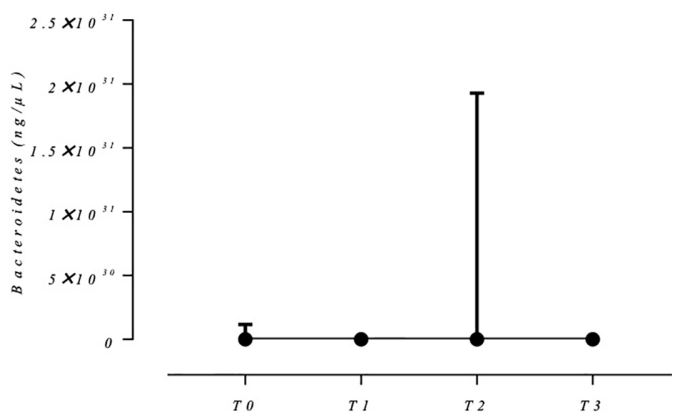

D

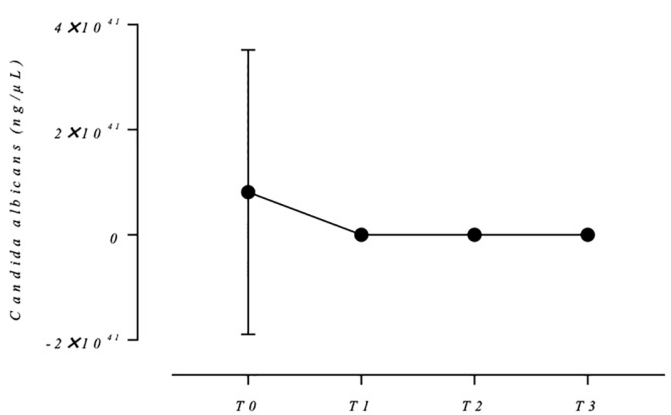

FIGURE 1 | Absolute quantification of total bacteria, Bacteroidetes and Firmicutes Phyla, and C. albicans from oral cavity of healthy patients submitted to impacted third molar surgery, after antiseptic and antibiotic intervention. T0: before surgery; T1: after 0.12\% chlorhexidine mouthwash, for 3 days; T2: after amoxicillin 875 mg, single dose, $1 \mathrm{~h}$ before surgery, followed by amoxicillin 875 mg every $12 \mathrm{~h}$, for 7 days after surgery; T3: 28 days after amoxicillin use. Statistical analyses were performed with a sample of 11 volunteers. $\eta p^{2}$ : Partial eta squared. Total bacteria, Bacteroidetes, and Firmicutes data were transformed by log 10 to achieve the RMANOVA premises. Candida albicans data were reciprocally transformed considering 1/x. (A) Median and interquartile range of total bacteria levels (ng/ $\mu$ l). RMANOVA statistics for total bacteria: $p$-value $=0.387$, power $=0.14, \eta p^{2}=0.100$. Significant $p$-value for Mauchly's test of sphericity required the GreenhouseGeisser correction. (B) Median and interquartile range of Bacteroidetes levels $(\mathrm{ng} / \mathrm{\mu l})$. RMANOVA statistics for Bacteroidetes: $p$-value $=0.396$, power $=0.16, \eta p^{2}=$ 0.119. Significant $p$-value for Mauchly's test of sphericity required the Greenhouse-Geisser correction. (C) Median and interquartile range of Firmicutes levels (ng/ $\mu$ l). RMANOVA statistics for Firmicutes: $p$-value $=0.000$, power $=1.00, \eta p^{2}=0.949$. Mauchly's sphericity was assumed. ${ }^{\star \star *}$ Means $p$-value lower than 0.0000 after Bonferroni adjustment for multiple comparisons. (D) Median and interquartile range of Candida albicans levels (ng/ul). RMANOVA statistics for Candida albicans: $p$-value $=0.101$, power $=0.51, \eta p^{2}=0.203$. Mauchly's sphericity was assumed

sample is the selection bias. To avoid that, only healthy patients, without previous infection and comorbidities, of a single dental office, operated by the same dentist were included. Furthermore, all patients received from researchers, the same brand of antiseptic and antibiotic for prophylaxis. In addition, the power of the test and the effect sizes were included. Noticeably, the statistical difference among the experimental phases regarding Firmicutes phylum level has a large power (1.00) and a large effect size (0.949) (Table 1).

Finally, our results showed that most patients seeking for third molar surgery had no presurgical infections, and even so received antiseptic and antibiotic prophylactic therapies. If no antibiotics were used for this group of patients, we could certainly contribute to a decrease in the resistance rates, as well as to diminish therapeutic failure in the treatment of infectious diseases (WORLD HEALTH ORGANIZATION, 2015). Investment in biosafety during the dental surgical procedures must be used as a tool to reduce antibiotics prescription to prevent infections.

\section{CONCLUSION}

The discussion about antibiotic usage in dentistry is not recent, and because of the lack of consensus, there is no safety protocol to avoid dysbiosis and antibiotic resistance. Further welldesigned studies involving microbiome sequencing are necessary to determine what species are suitable to selection during antiseptic and antibiotic therapy. In spite of not identifying these species, our preliminary results warn about the need of personalized tools and protocols in dentistry, targeting individual particular requirements, avoiding the use of a generalized predetermined protocol. 


\section{PRIOR PRESENTATION}

Partial results of this study were presented at the 31th ECCMID, Vienna, Austria, 2021.

\section{DATA AVAILABILITY STATEMENT}

The original contributions presented in the study are included in the article/supplementary material. Further inquiries can be directed to the corresponding author.

\section{ETHICS STATEMENT}

The studies involving human participants were reviewed and approved by Universidade São Francisco. The patients/ participants provided their written informed consent to participate in this study.

\section{REFERENCES}

Beier, R. C., Byrd, J. A., Andrews, K., Caldwell, D., Crippen, T. L., Anderson, R. C., et al. (2021). Disinfectant and Antimicrobial Susceptibility Studies of the Foodborne Pathogen Campylobacter Jejuni Isolated From the Litter of Broiler Chicken Houses. Poult. Sci. 100 (2), 1024-1033. doi: 10.1016/ j.psj.2020.10.045

Coulthard, P., Bailey, E., Esposito, M., Furness, S., Renton, T. F., and Worthington, H. V. (2014). Surgical Techniques for the Removal of Mandibular Wisdom Teeth. Cochrane Database Syst. Rev. 7, CD004345. doi: 10.1002/14651858. CD004345

Dallaserra, M., Poblete, F., Vergara, C., Cortés, R., Araya, I., Yanine, N., et al. (2020). Infectious Postoperative Complications in Oral Surgery. An Observational Study. J. Clin. Exp. Dent. 12 (1), e65-e70. doi: 10.4317/ medoral.55982

Garratt, I., Aranega-Bou, P., Sutton, J. M., Moore, G., and Wand, M. E. (2021). Long-Term Exposure to Octenidine in a Simulated Sink Trap Environment Results in Selection of Pseudomonas Aeruginosa, Citrobacter, and Enterobacter Isolates With Mutations in Efflux Pump Regulators. Appl. Environ. Microbiol. 87 (10), e00210-e00221. doi: 10.1128/AEM.00210-21

Guo, X., Xia, X., Tang, R., Zhou, J., Zhao, H., and Wang, K. (2008). Development of a Real-Time PCR Method for Firmicutes and Bacteroidetes in Faeces and its Application to Quantify Intestinal Population of Obese and Lean Pigs. Lett. Appl. Microbiol. 47 (5), 367-373. doi: 10.1111/j.1472-765X.2008. 02408.x

Khalil, D., Hultin, M., Rashid, M. U., and Lund, B. (2016). Oral Microflora and Selection of Resistance After a Single Dose of Amoxicillin. Clin. Microbiol. Infect. 22 (11), 949.e1-949.e4. doi: 10.1016/j.cmi.2016.08.008

Kirk, D. G., Liston, P. N., Tong, D. C., and Love, R. M. (2007). Influence of Two Different Flap Designs on Incidence of Pain, Swelling, Trismus, and Alveolar Osteitis in the Week Following Third Molar Surgery. Oral. Surg. Oral. Med. Oral. Pathol. Oral. Radiol. Endod. 104 (1), e1-e6. doi: 10.1016/ j.tripleo.2007.01.032

Lacasa, J. M., Jiménez, J. A., Ferrás, V., Bossom, M., Sóla-Morales, O., García-Rey, C., et al. (2007). Prophylaxis Versus Pre-Emptive Treatment for Infective and Inflammatory Complications of Surgical Third Molar Removal: A Randomized, Double-Blind, Placebo-Controlled, Clinical Trial With Sustained Release Amoxicillin/Clavulanic Acid (1000/62.5 Mg). Int. J. Oral. Maxillofac. Surg. 36 (4), 321-327. doi: 10.1016/j.ijom.2006.11.007

Lindeboom, J. A. (2008). The Controversy Continues! Int. J. Oral. Maxillofac. Surg. 37 (2), 199-200. doi: 10.1016/j.ijom.2007.07.027

Lodi, G., Azzi, L., Varoni, E. M., Pentenero, M., Del Fabbro, M., Carrassi, A., et al. (2021). Antibiotics to Prevent Complications Following Tooth Extractions.

\section{AUTHOR CONTRIBUTIONS}

$\mathrm{CN}$ and CA contributed with study design, conduction of experiments, data analysis, and writing of the manuscript. NR, KFR, KLR, and AP contributed for conduction of experiments. $\mathrm{TP}$ contributed with data analysis and writing and revision of the manuscript. RG contributed with study design, conduction of experiments, data analysis, and writing and revision of the manuscript. All authors contributed to the article and approved the submitted version.

\section{ACKNOWLEDGMENTS}

We would like to thank National Council for Scientific and Technological Development (CNPq), Ministry of Science and Technology, Brazil, and by The São Paulo State Research Foundation (FAPESP) and Universidade São Francisco, for supporting in our researchers.

Cochrane Database Syst. Rev. 2, CD003811. doi: 10.1002/14651858. CD003811.pub3

Luaces-Rey, R., Arenaz-Búa, J., Lopez-Cedrun-Cembranos, J. L., Martínez-Roca, C., Pértega-Díaz, S., and Sironvalle-Soliva, S. (2010). Efficacy and Safety Comparison of Two Amoxicillin Administration Schedules After Third Molar Removal. A Randomized, Double-Blind and Controlled Clinical Trial. Med. Oral. Patol. Oral. Cir. Bucal. 15 (4), e633-e638. doi: 10.4317/ medoral.15.e633

Menon, R. K., Kar Yan, L., Gopinath, D., and Botelho, M. G. (2019). Is There a Need for Postoperative Antibiotics After Third Molar Surgery? A 5-Year Retrospective Study. J. Investig. Clin. Dent. 10 (4), e12460. doi: 10.1111/ jicd. 12460

Merchel Piovesan Pereira, B., Wang, X., and Tagkopoulos, I. (2021). BiocideInduced Emergence of Antibiotic Resistance in Escherichia Coli. Front. Microbiol. 12, 640923. doi: 10.3389/fmicb.2021.640923

Nadkarni, M. A., Martin, F. E., Jacques, N. A., and Hunter, N. (2002). Determination of Bacterial Load by Real-Time PCR Using a Broad-Range (Universal) Probe and Primers Set. Microbiology (Reading) 148 (Pt 1), 257266. doi: 10.1099/00221287-148-1-257

Neves, C. A., Alves, C. H., Rocha, N. C., Russi, K. L., Rizzardi, K. F., Parisotto, T. M., et al. (2020). Uninfected Antibiotics and Third Molars: Are You Contributing to Resistance? J. Infect. Control 9 (3), 196-201.

Plachokova, A. S., Andreu-Sánchez, S., Noz, M. P., Fu, J., and Riksen, N. P. (2021). Oral Microbiome in Relation to Periodontitis Severity and Systemic Inflammation. Int. J. Mol. Sci. 22 (11), 5876. doi: 10.3390/ijms22115876

Rahn, S., Schuck, A., Kondakci, M., Haas, R., Neuhausen, N., Pfeffer, K., et al (2016). A Novel Comprehensive Set of Fungal Real Time PCR Assays (fuPCR) for the Detection of Fungi in Immunocompromised Haematological PatientsA Pilot Study. Int. J. Med. Microbiol. 306 (8), 611-623. doi: 10.1016/ j.ijmm.2016.10.003

Riba-Terés, N., Jorba-García, A., Toledano-Serrabona, J., Aguilar-Durán, L., Figueiredo, R., and Valmaseda-Castellón, E. (2021). Microbiota of Alveolar Osteitis After Permanent Tooth Extractions: A Systematic Review. J. Stomatol. Oral. Maxillofac. Surg. 122 (2), 173-181. doi: 10.1016/j.jormas.2020.08.007

Rizzardi, K. F., Indiani, CMDSP, Mattos-Graner, R. O., de Sousa, E. T., NobreDos-Santos, M., and Parisotto, T. M. (2021). Firmicutes Levels in the Mouth Reflect the Gut Condition With Respect to Obesity and Early Childhood Caries. Front. Cell Infect. Microbiol. 11, 593734. doi: 10.3389/fcimb. 2021.593734

Robertson, D., and Smith, A. J. (2009). The Microbiology of the Acute Dental Abscess. J. Med. Microbiol. 58 (Pt 2), 155-162. doi: 10.1099/jmm.0.003517-0

Russi, K. L., Neves, C. A., Lima, L. P., Siqueira, C. S., and Girardello, R. (2020). “Avaliação do Uso De Antissépticos Bucais Por Indivíduos Saudáveis," in 
XXVI Encontro De Pós-Graduação E Iniciação Científica (Bragança Paulista, Brasil: Universidade São Francisco).

Samal, M., Karny, M., Benali, H., Backfrieder, W., Todd-Pokropek, A., and Bergmann, H. (1999). Experimental Comparison of Data Transformation Procedures for Analysis of Principal Components. Phys. Med. Biol. 44 (11), 2821-2834. doi: 10.1088/0031-9155/44/11/310

Silvestri, A. R. Jr., and Singh, I. (2003). The Unresolved Problem of the Third Molar: Would People Be Better Off Without It? J. Am. Dent. Assoc. 134 (4), 450-455. doi: 10.14219/jada.archive.2003.0194

Stein, K., Farmer, J., Singhal, S., Marra, F., Sutherland, S., and Quiñonez, C. (2018). The Use and Misuse of Antibiotics in Dentistry: A Scoping Review. J. Am. Dent. Assoc. 149 (10), 869-884.e5. doi: 10.1016/j.adaj.2018.05.034

Sukegawa, S., Yokota, K., Kanno, T., Manabe, Y., Sukegawa-Takahashi, Y., Masui, M., et al (2019). What Are the Risk Factors for Postoperative Infections of Third Molar Extraction Surgery: A Retrospective Clinical Study? Med. Oral. Patol. Oral. Cir. Bucal. 24 (1), e123-e129. doi: 10.4317/medoral.22556

WORLD HEALTH ORGANIZATION. (2015). Global Action Plan on Antimicrobial Resistance. Available at: https://www.who.int/publications/i/ item/global-action-plan-on-antimicrobial-resistance.

Yoshida, K., Kodama, Y., Nishikawa, A., Estacio Salazar, A. R., Toyama, A., and Takagi, R. (2021). Comparison Between the Prophylactic Effects of Amoxicillin
24 and 48 Hours Pre-Operatively on Surgical Site Infections in Japanese Patients With Impacted Mandibular Third Molars: A Prospective Cohort Study. J. Infect. Chemother. 27 (6), 845-851. doi: 10.1016/j.jiac.2021.01.018

Conflict of Interest: The authors declare that the research was conducted in the absence of any commercial or financial relationships that could be construed as a potential conflict of interest.

Publisher's Note: All claims expressed in this article are solely those of the authors and do not necessarily represent those of their affiliated organizations, or those of the publisher, the editors and the reviewers. Any product that may be evaluated in this article, or claim that may be made by its manufacturer, is not guaranteed or endorsed by the publisher.

Copyright (C) 2021 Neves, Alves, Rocha, Rizzardi, Russi, Palazzi, Parisotto and Girardello. This is an open-access article distributed under the terms of the Creative Commons Attribution License (CC BY). The use, distribution or reproduction in other forums is permitted, provided the original author(s) and the copyright owner(s) are credited and that the original publication in this journal is cited, in accordance with accepted academic practice. No use, distribution or reproduction is permitted which does not comply with these terms. 